\title{
Pure study and experimental application of laser measurement for students in independent colleges
}

\section{Wanyi Zhang, Zhe Liu}

Wanyi Zhang, Zhe Liu, "Pure study and experimental application of laser measurement for students in independent colleges," Proc. SPIE 10452, 14th Conference on Education and Training in Optics and Photonics: ETOP 2017, 1045251 (16 August 2017); doi: 10.1117/12.2269596

EDent: 14th Conference on Education and Training in Optics and Photonics, ETOP 2017, 2017, Hangzhou, China 


\title{
Pure study and experimental application of laser measurement for students in independent colleges
}

\author{
Wanyi Zhang*a ,Zhe Liu ${ }^{\mathrm{b}}$ \\ College of optical and electronic information, Changchun University of Science and Technology \\ 399,Daxin Road,Hi-Tech Development Zone ,Changchun City, Jilin Province, China,P.C130012.
}

\begin{abstract}
The cultivation of independent college students is the development of applied talents and the strength of students' innovative ability. This requires teachers to make better use of the resources of the school, to develop students' ability with greatest possible and to encourage students to learn independently and personality development. We can carry out multi-discipline curriculum design practice after the study of related disciplines in order to make students have a more in-depth understanding and learning of the professional courses. In this paper, we will research on curriculum design based on the combination of Laser Measurement Technology and Digital Image Processing.
\end{abstract}

Key words: independent college, multi-discipline, laser measurement technology, digital image processing

\section{INTRODUCTION}

Laser Measurement Technology is a professional required course for junior students of Measurement and Control Technology and Instrument Specialty and a professional elective course for senior students of Optoelectronic Information Engineering Specialty in Optoelectronic Engineering Branch. This course has its special significance, the development of photoelectric technology and laser measuring technology is closely related, with the development of optoelectronic devices, such as photocells, photodiodes and CCD camera device, the laser and photoelectric testing technology, online detection and control technology, optical fiber sensor technology and visual detection technology is more common development and application. Digital Image Processing is also a major required course for major students of Measurement and Control Technology and Instrument Specialty, the two courses are studied separately in the two semesters, and the integrated curriculum design is carried out just after the completion of Digital Image Processing courses, in order to strengthen the practical application of the two disciplines, at the same time to play the role of review and in-depth.

\section{THE IMPORTANCE OF LASER MEASUREMENT TECHNOLOGY}

The Laser Measuring Technology has some outstanding advantages: it is contacted with the light directly so as to achieve high precision measurement; photoelectric conversion is very easy to do with a computer, realize the automatic analysis, processing, display and print of the measurement data. In this paper, we mainly discuss the combined application of digital holographic measurement experiment in Laser Measuring Technology and Digital Image Processing.

\subsection{The basic principle of digital holography ${ }^{\text {[11 }}$}

The basic principle of digital holography is the same as traditional optical holography, they all need two processes: recording and reconstruction. But in the process of recording, storage and reconstruction of holograms, different methods have been adopted to realize it. The recording element used in digital holography is electronic photosensitive device $\mathrm{CCD}$, and storage device is high-speed computer. Digital holography can use the computer to optical simulate the reconstruction process directly. We can use the off-axis optical path shoot hologram to obtain the three-dimensional reconstruction of diffuse objects, at the same time, it is possible to obtain some fringes which may be covered by the three-dimensional reconstruction or in the nearby location. These fringes represent information about displacement, deformation or rotation of an object.

397964022@qq.com; phone13394300310

14th Conference on Education and Training in Optics and Photonics: ETOP 2017, edited by Xu Liu,

Xi-Cheng Zhang, Proc. of SPIE Vol. 10452, 1045251 - @ 2017 ICO, IEEE, OSA, SPIE

CCC code: $0277-786 X / 17 / \$ 18 \cdot$ doi: $10.1117 / 12.2269596$ 


\subsection{Experimental process}

The experimental setup of optical system is mainly composed of the following parts: laser, collimating and beam expanding system, attenuator, beam splitter, imaging lens, CCD, image acquisition card, electro addressed liquid crystal (EALCD), etc. The collimating beam expanding system is composed of a micro objective lens, a pinhole and a collimating objective lens.



Figure 2.1 Fresnel digital holographic recording device figure

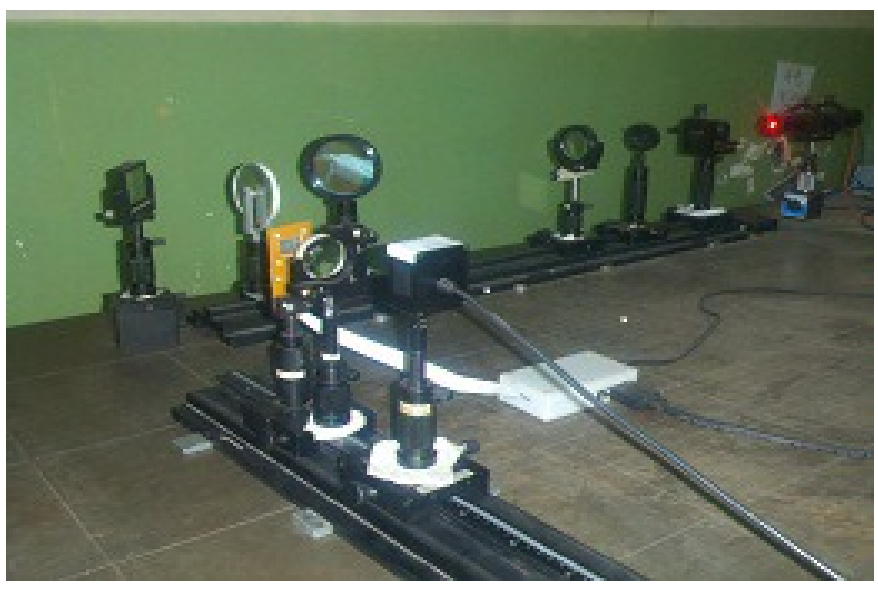

Figure 2.2 Fresnel digital holographic reconstruction device figure

\subsection{Experimental results}

The interference fringe of figure 2.5 represents the displacement or deformation information of the experiment object, but the fringe clarity is not enough due to the limited experimental conditions, so we can not completely judge the effective information, it need for subsequent processing. 


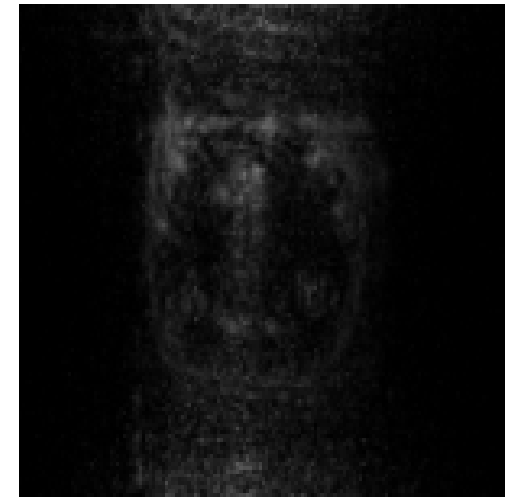

Figure2.3 Reconstructed image before displacement

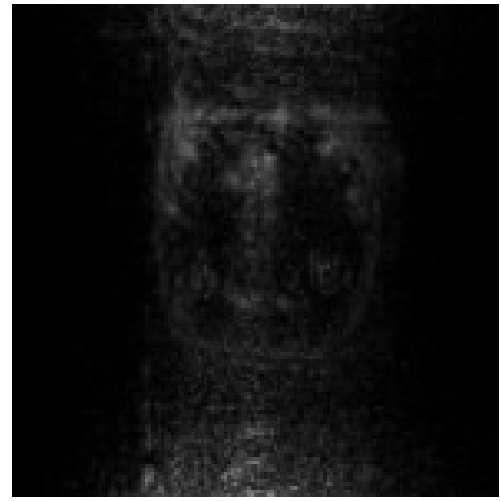

Figure2.4 Reconstructed image after displacement



Figure2.5 Interference fringe pattern

\section{APPLICATION OF DIGITAL IMAGE PROCESSING IN HOLOGRAPHIC INTERFEROMETRY}

\subsection{The purpose of digital image processing}

1. To improve the image quality by visual sense to improve the visual effect or to achieve the image requirements of the receiving system, such as the brightness of images or color transform is used to enhance or inhibit certain ingredients, it can also take geometric transform method to the image.

2 To obtain some features or special information in the image, which provides convenience for computer analysis of images. Essentially, the process of extraction is a preprocessing for pattern recognition and computer vision. The extracted information can be gray feature, color feature, frequency domain feature, regional feature, shape feature, boundary feature, texture feature, topological feature and relational structure, etc.

3 To facilitate the storage and transmission of the image, the image data must be encoded, transformed and compressed, etc.

\subsection{Advantages of digital image processing}

1 There is an essential difference between the digital processing and the simulation processing of the image. The quality of the reconstruction image is degraded due to the operation of storage, replication and transmission of the simulation processing, but the reconstruction image runs through the whole process of the digital processing, so the original image has a high accuracy of reconstruction.

2 High precision of image processing. If the image is simulated, accuracy increased by an order of magnitude need the greatly improved of the processing device and the cost is high. However, if an image is digitized, the accuracy of the digital image can be satisfied with the applying of current device. 
3 Wide range of image processing, it can be derived from a variety of information sources. Range from visible to invisible. The image is transformed into a digital form, and can be processed by a computer. Digital image processing technology is suitable for any kind of image, as long as the corresponding image information acquisition measures for different image information sources.

\subsection{Common digital image processing methods ${ }^{\mathrm{t} 2 \mathrm{I}}$}

\subsubsection{Laplace Gauss operator algorithm}

From the mathematical analysis, the essence of image blurring is due to the influence of the average or integral operation. If the image is under the inverse operation, such as the use of differential operators, you can make the image becomes clear. Gradient is one of the commonly used differential methods. The gradient is proportional to the rate of change of the gray level, when the gray level is constant, the gradient is zero.

However, the first differential is far away from the second differential. In this experiment, the image preprocessing is used in second linear differential molecular - Laplacian operator.

The Laplacian operator has the isotropy and rotation invariance of the filter. There are same results between filtering after the rotation of the original image and rotation after the filtering, so it has a good effect for the point, line, boundary extraction of the image.

Using Laplacian operator to preprocess the holographic image with MATLAB programming, the original object is as shown in Figure 3.1, the untreated Fresnel hologram and the reconstructed image are shown in figures 3.2 and 3.3, the hologram and the reconstructed image processed by Laplacian operator are shown in Figure 3.4 and 3.5. It can be seen that the quality of reconstructed image is significantly improved compared with that of the original image after preprocessing by Laplacian operator.

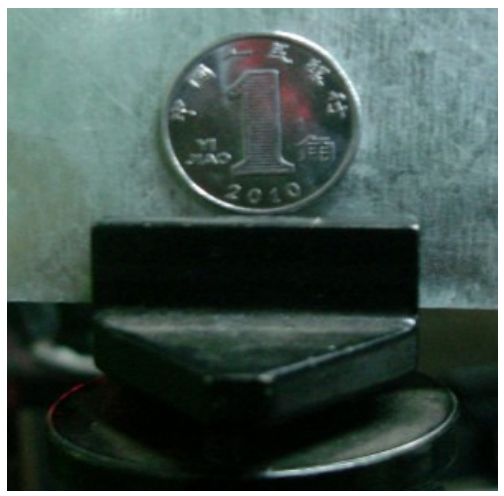

Figure3.1 Experimental object

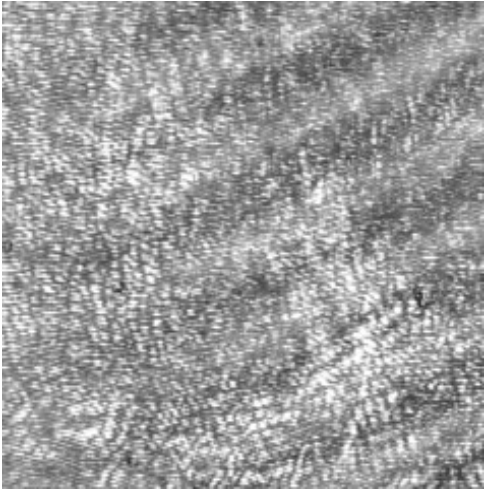

Figure3.2 Unprocessed hologram

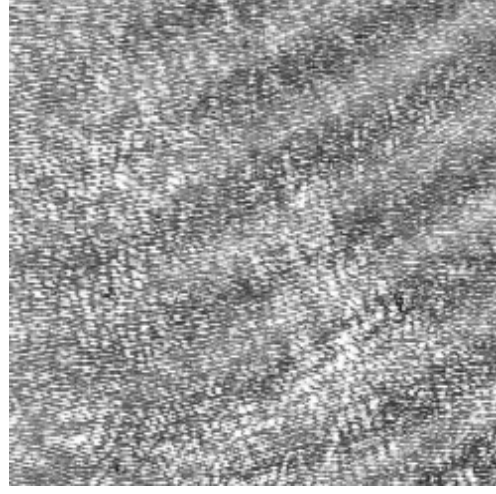

Figure3.3 Processed hologram



Figure3.4 Unprocessed reconstructed image

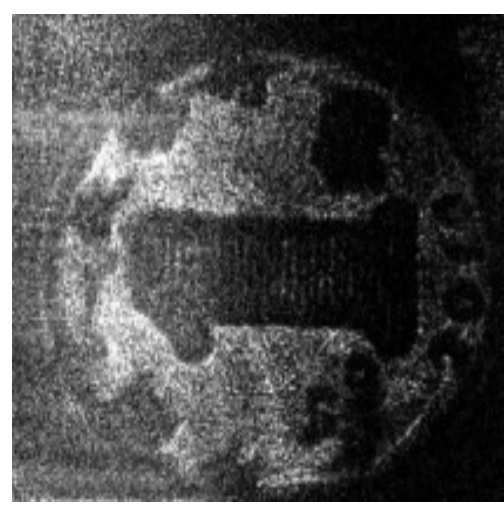

Figure3.5 Processed reconstructed image 


\subsubsection{Median filtering}

Median filtering method for image processing is a nonlinear smoothing, it can eliminate salt and pepper noise effectively and has special effect on phase analysis of fringe image in optical measurement, it often used for protecting edge information, so it is the classic method of noise smoothing.

The gray value of each pixel is equal to the value of the gray value of all pixels in the neighborhood window, the value of one point is replaced by the median of each sample in a neighborhood of the point in a digital image or sequence, make sure the surrounding pixel value is close to the true value, thus the isolated noise points are eliminated. The number of data to be processed by sorting out the odd number of data from a sampling window in the image or replacing the data with the median after the sorting.

\subsubsection{Homomorphic filtering}

Homomorphic filtering of the image is a special class of nonlinear processing which according to the generalized superposition principle, it is showed with the linear transformation between the vector space of the input and output in algebra, the range of the image brightness is compressed and the image contrast is enhanced in the in the frequency domain. Its basic idea is to transform the nonlinear problem into a linear problem. First, to do some mathematical calculation of nonlinear hybrid signal into additive, so that you can process image with linear filtering method, and finally do the inverse operation of corresponding mathematical operations, then recover the image.

After homomorphic filtering using MATLAB software program, the area with lower image brightness becomes visible and the highlight area becomes clearer, the contrast and the image visibility increases.

\subsubsection{The combine of Gray transform and Wiener filtering}

The gray level transformation is to change the gray value of pixels in the original image according to a certain transformation relation in order to achieve some objective conditions. The contrast can be improved by gray-scale transformation of image, a specific gray enhancement method is determined when he gray transformation relation is determined.

Due to various factors such as the system, often resulting in uneven image, contrast is not enough, thus affecting the visual effect of the human eye to see the image during the photoelectric conversion. Through the image acquisition system in the image pixel processing, we can make the whole image uniformity.

Wiener filter is a linear smoothing filter which has the function of adaptive filtering for digital image, the output of the filter can be adjusted according to the region variance of the image. In this experiment, the MATLAB software is used to write the program and the interference fringe image is processed by the method of gray level transform and Wiener filtering to improve the fringe contrast.

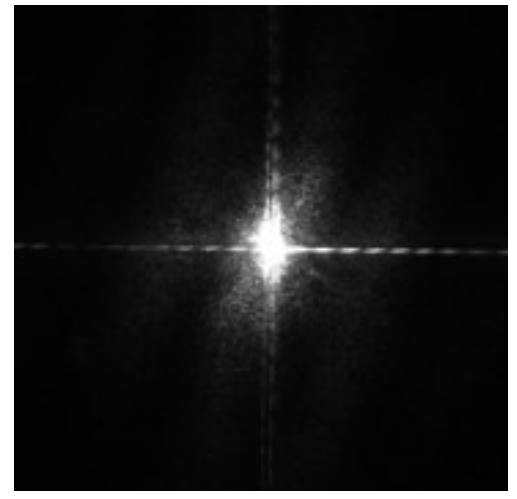

Figure3.6 Unprocessed fringe pattern

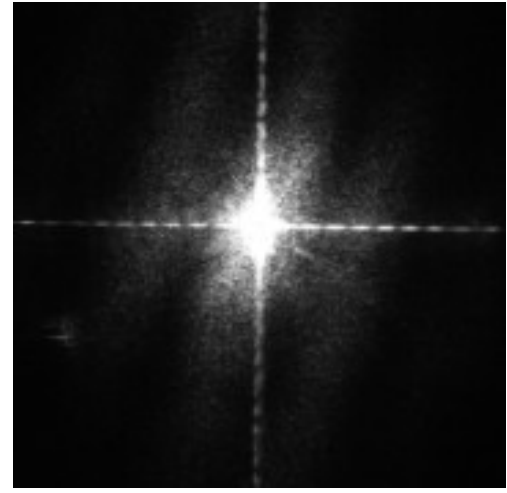

Figure3.7 Fringe pattern after median filtering 

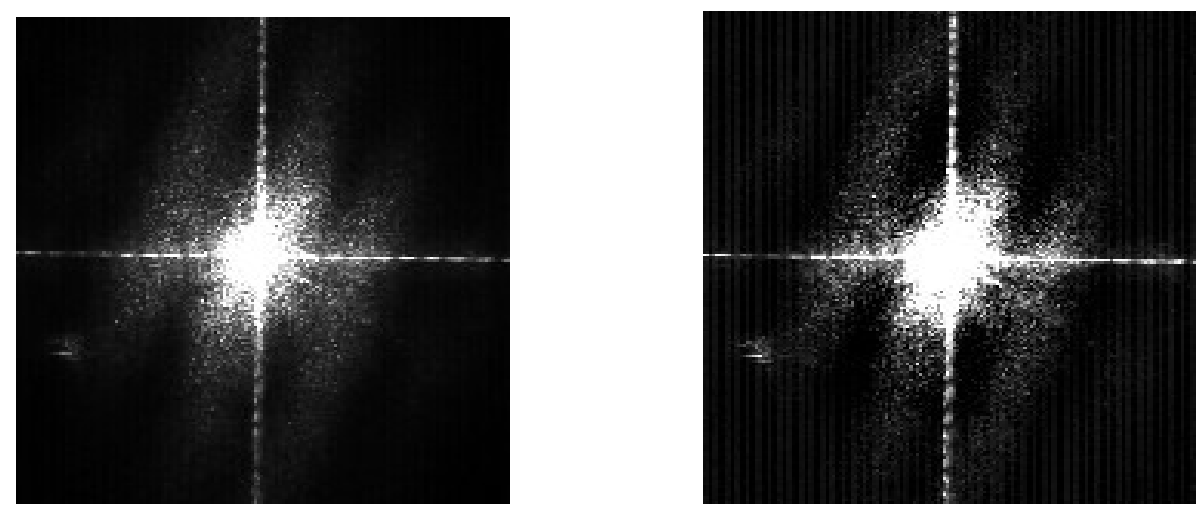

Figure3.8 Fringe pattern after gray transform and Wiener filtering Figure3.9 Fringe pattern after homomorphic processing

Three different digital image processing methods are used for the interference fringe pattern, unprocessed fringe pattern is as shown in Figure 3.6, fringe pattern after median filtering is as shown in Figure 3.7, Fringe pattern after gray transform and Wiener filtering is as shown in Figure 3.8, fringe pattern after homomorphic processing is as shown in Figure 3.9. It can be seen that the fringe pattern after the digital image processing has improved the clarity and contrast of the fringe pattern.

\section{GROUPED CURRICULUM DESIGN LEARNING}

The students can enter into the stage of curriculum design after the two courses are learned completely. For example, a class of 30 students can be divided into five groups, each group of six students, among them, three students for the early laser measurement experiment and get a clear picture of experiment through the repeated experimental process, another three students to carry out digital image processing software process. The entire curriculum design takes about three weeks, it can be reasonable allocation of time. Finally, the image quality is evaluated, image quality evaluate is divided into objective evaluation and subjective evaluation, objective evaluation method is the use of a test card, and subjective evaluation is the use of visual observation and subjective evaluation of image quality.

Two points need to pay attention: First, there are a lot of measurement methods in the Laser Measurement Technology. In this paper, holographic interferometry is just one of the methods, the students can choose other measurement methods according to their own expertise to get the experimental pictures. Second, the digital image processing method in the paper is also just enumerated, in practice, the students also need to carry on the concrete analysis to carry on the programming and the application according to the actual situation.

The three week curriculum design can make the students have a more in-depth understanding of the two disciplines, hands-on ability will be greatly improved, this is also the direction of independent college students' learning and training -- the cultivation of applied talents.

\section{REFERENCES}

[1] Booth, Wensheng Wang., [Contemporary Optical Measurement Technology], China Machine Press, Beijing, 116-130 (2013).

[2] Booth, Jianhua Xue., [Image processing and analysis ], China Science Press, Beijing, 73-80 (1992). 